\title{
Emerging Drugs in Dermatology
}

\section{Janet Nzisa}

Editorial Assistant

Citation: EMJ Dermatol. 2021;9[1]:29-33.
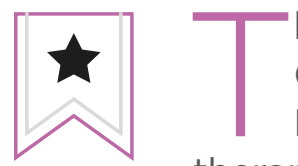

he ANNUAL European Academy of Dermatology and Venereology (EADV) Virtual Congress 2021 featured the latest updates on emerging drugs in dermatology.

Presentations covered a wide range of topics such as immunotherapy versus targeted therapy in melanoma, targeting cytokine pathways in psoriasis, emerging biologics, and the promise of personalised medicine in dermatology.

\section{IMMUNOTHERAPY VERSUS TARGETED THERAPY IN MELANOMA}

Melanoma is a prominent type of skin cancer and treatment varies depending on the mutation and malignancy of the disease. The overall survival (OS) rate of metastatic melanoma is $35-50 \%$. Pablo Fernandez-Peñas, Westmead Hospital, Sydney Medical School, Australia, opened the session by stating the discussion was based on BRAF-mutated melanoma. FernandezPeñas attested that targeted therapies are not beneficial for BRAF wild-type melanoma. The two approaches in treatments currently used in metastatic melanoma are adjuvant and neoadjuvant treatment. According to recent data on non-resectable melanoma, the advanced stage of metastatic melanoma, immunotherapies supposedly have a long-term survival benefit. The data presented by Fernandez-Peñas on ipilimumab and nivolumab displayed a 5-year OS rate of $52 \%$. He compared these data to targeted therapy using dabrafenib and trametinib, which showed an OS rate of $34 \%$ in 5 years. A meta regression analysis comparing both targeted therapies and immunotherapy drugs demonstrated that a combination of ipilimumab and nivolumab has a statistically significant greater OS rate compared to dabrafenib and trametinib. Additionally, results of an ongoing meta-analysis carried out on five randomised clinical trials showed that a combination of targeted and immunotherapy, known as triple therapy, could be effective in the treatment of metastatic melanoma, but could cause increased toxicity.

In conclusion, Fernandez-Peñas confirmed that immunotherapy as a first-line melanoma treatment had a superior long-term outcome. However, targeted therapies had rapid symptom control and higher chance of short-term response. Similar results have been observed in Stage III/IV melanoma with adjuvant therapy; however, there have been limitations in the current research, and further data need to be analysed to confirm these results. There are more novel targeted and immunotherapies in the horizon in Phase I and II stages of research. Other newer therapies for melanoma include viral vectors (e.g., T-VEC), T-cell therapies (e.g., CAR-T cells), and intratumoural therapies (e.g., TLR9). 


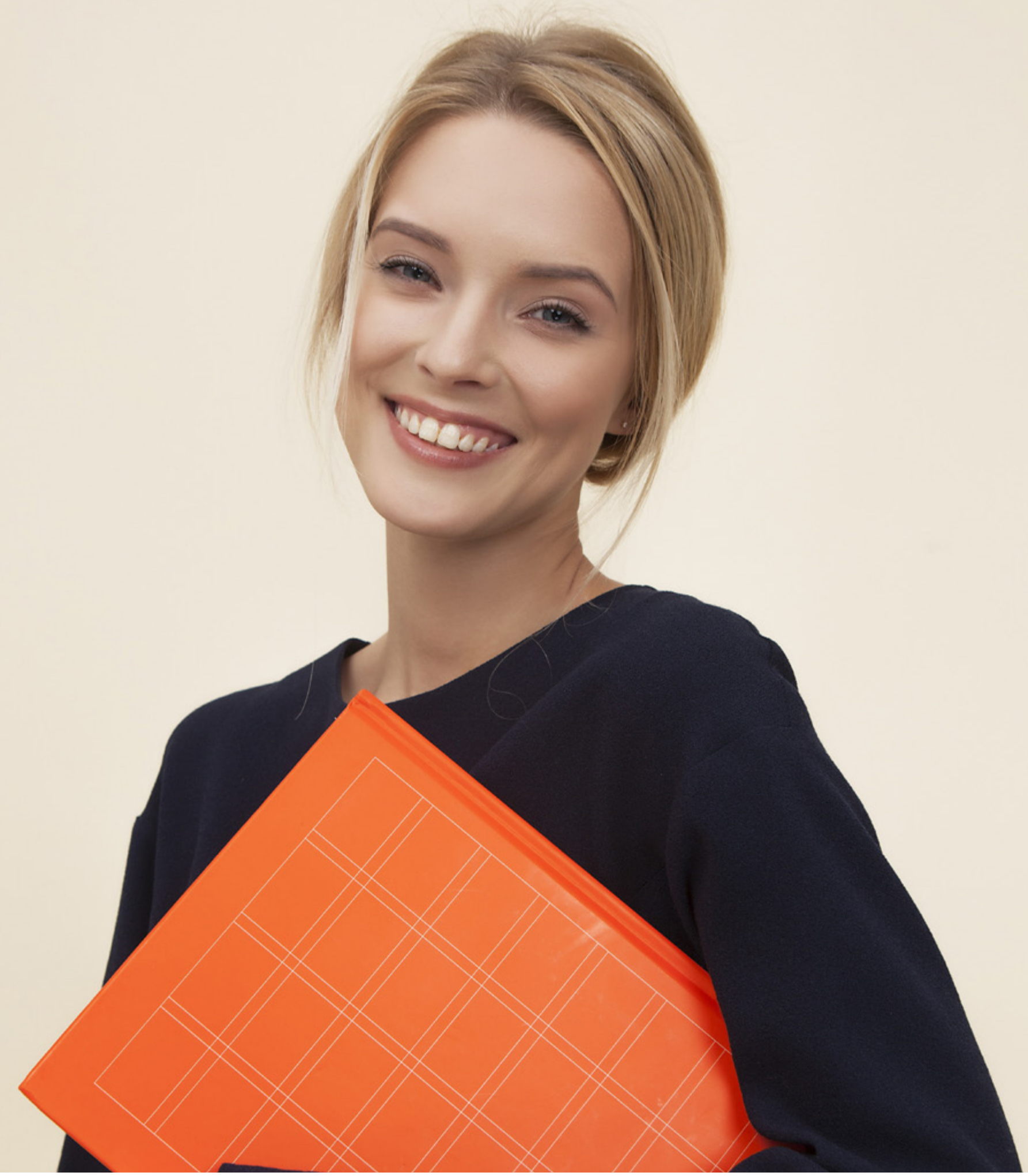

\section{TARGETING CYTOKINE PATHWAYS IN PSORIASIS}

Kamran Ghoreschi, Department of Dermatology, Venereology, and Allergology Charité Universitätsmedizin Berlin, Germany, began the session by stating that the immunology and genetic associations of psoriasis are wellunderstood. He presented emerging data and drugs for psoriasis that focused on IL-17 and IL23 inhibitors. Bimekizumab was approved in 2021 and is an anti-IL17A, anti-IL-17F, and anti-IL17AF monoclonal antibody. Ghoreschi confirmed that bimekizumab had been tested against other established anti-psoriatic drugs such as secukinumab, adalimumab, and ustekinumab. The results show that bimekizumab efficacy is superior to these drugs, with greater clearance of skin compared to secukinumab and ustekinumab. Ghoreschi attested that it may be useful to switch from one IL-17 inhibitor to another, as some patients may lose their primary response after a 
period of exposure to one IL-17 inhibitor. He shared an example on the advisable sequence for IL-17 inhibitors: initial treatment with secukinumab; if a patient loses the primary response after 1-2 years, the healthcare professional should switch to ixekizumab or bimekizumab. Furthermore, there are novel emerging IL-17 inhibitors in the horizon such as netakimab (anti-IL-17A), CJM112 (anti-IL-17A/F), and sonelokimab (anti-IL-17A).

Finally, Ghoreschi shared recently published data on COVID-19 vaccination with antipsoriatic treatment. The data involved patients currently using targeted biological monotherapy, methotrexate, versus healthy control subjects. Immunogenicity was evaluated before and on Day $28( \pm 2)$ after the BNT162b2 vaccine to analyse the spike-specific T-cell responses. The results showed neutralising activity against the wild-type severe acute respiratory syndrome coronavirus 2 was significantly lower in patients receiving methotrexate (median 50\% inhibitory dilution $\left[\mathrm{ID}_{50}\right]$ : 129) compared to the controls $\left(\mathrm{ID}_{50}\right.$ : 317 [interquartile range: 213-487]; $\mathrm{p}=0.0032$ ); however, it was preserved in patients receiving targeted biological monotherapy $\left(\mathrm{ID}_{50}: 269\right.$ [interquartile range: 141-418]). Cellular immune responses were induced in all groups and were not attenuated in patients receiving targeted biological monotherapy or methotrexate compared to the controls.

\section{EMERGING BIOLOGICS AND SMALL MOLECULES FOR ATOPIC DERMATITIS}

Atopic dermatitis (AD) is a common, chronic inflammatory disease. Richard Langley, Division of Clinical Dermatology and Cutaneous Science, Dalhousie University, Nova Scotia, Canada, began the presentation with a case study of a patient with wide-spread dermatitis. According to Langley, the patient would flare up and use steroids as treatment and, once the flares cleared up, he would stop the treatment and the flare would appear again; it was a vicious cycle. Langley stated that there was an unmet need for acute and long-term control in $A D$. The understanding of pathophysiology of dermatitis is evolving and therefore there is potential for fulfilling this unmet need. The two groups of emerging systemic therapies and biologics discussed by Langley were cytokine antagonists dupilumab (IL-4 and IL-13) and small molecules/JAK inhibitors (abrocitinib, baricitinib, and upadacitinib). There are ongoing Phase III trials with dupilumab for adult patients with moderate-severe AD. However, side effects have been observed such as nasopharyngitis, upper respiratory tract infections, and conjunctivitis.

Langley emphasised that JAK inhibitors by nature cannot be selective for a particular JAK; instead, they show preferential activity for certain
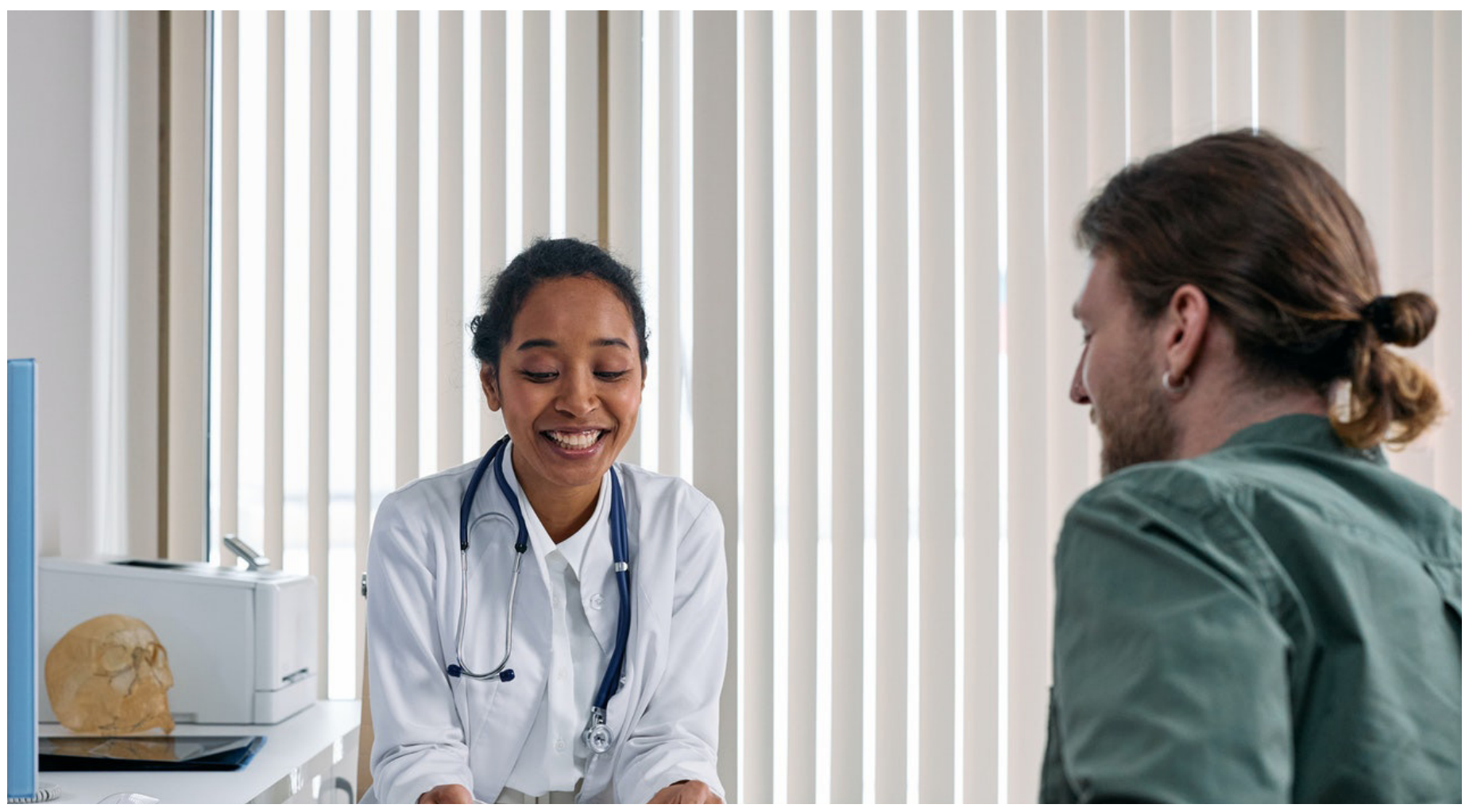


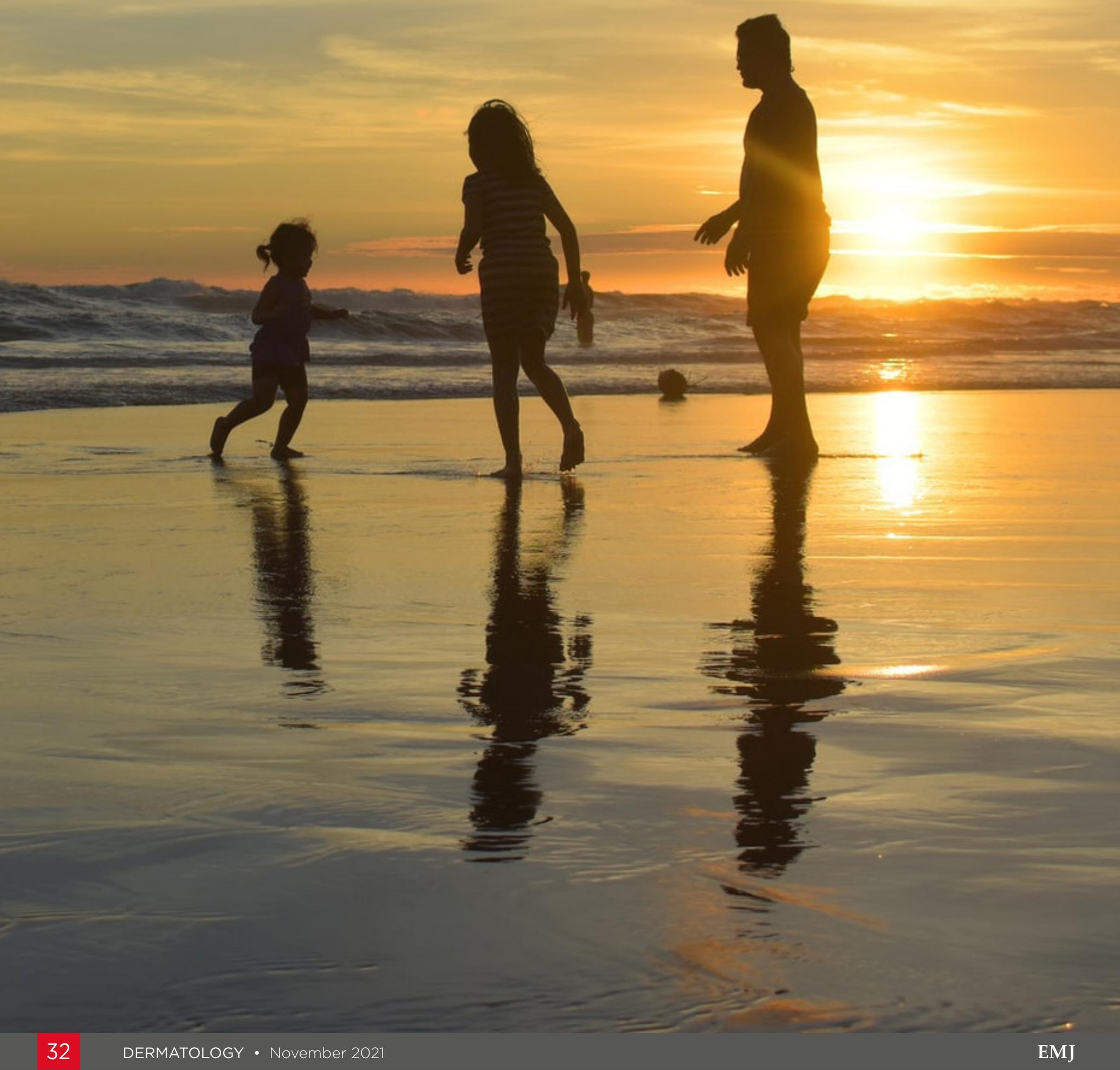


JAKs over others, although at sufficiently high concentrations they would likely inhibit all the JAKs. Abrocitinib shows higher selectivity for JAK 1 and 2; both baricitinib and upadacitinib have a higher selectivity for JAK 1. Results of a study for treatment with baricitinib $2 \mathrm{mg}$ show a $75 \%$ reduction in Eczema Area and Severity Index (EASI-75) in patients' response at Week 16, 32, and 52 of $40 \%, 51 \%$, and $49 \%$, respectively. The safety profile of baricitinib was reassuring, with low rates of serious infections. Another study presented by Langley on the efficacy of abrocitinib (200 mg) plus a topical therapy among adolescents with moderate $A D$ showed a faster response than dupilumab. Langley confirmed that the utilisation of this drug may be influenced by the desire for quicker responses and greater clearance of $A D$; however, tolerability issues should be considered. Langley emphasised the importance of the novel data and stated that the current results have been promising. However, the present data are shortterm and long-term data would be beneficial in determining a treatment paradigm for acute AD.

\section{THE PROMISE OF PERSONALISED MEDICINE}

Nicholas Reynolds, Dermatology and Director of Diagnostics, Newcastle University, UK, and Royal Victoria Infirmary, Newcastle upon Tyne,
UK, started his session by attesting that the efficacy of therapeutic agents is variable and largely unpredictable at times, with both primary and secondary failure. However, in cancer the genetic basis of the disorder has led to welldefined stratified medicine that has been adopted into clinical practise. According to Reynolds, this is harder in complex inflammatory disorders to progress well-defined biological treatments, although there have been some recent advancements. Using biomarker profile analysis, researchers can predict responders, non-responders, and patients who may develop adverse effects following a treatment. A 2016 psoriasis recommendation from the World Health Organization (WHO) stated that all patients should have access to comprehensive, individually adapted treatment; additionally, research on new treatments should focus on options that can be available globally. The Psoriasis Stratification to Optimise Relevant Therapy (PSORT) consortium are currently attempting to stratify biological therapies for patients with moderate-severe psoriasis. Reynolds emphasised the concept of 'P4 medicine', meaning predictive, preventative, personalised, and participatory medicine, which is widely used in clinical practice and would be highly beneficial to dermatology. In conclusion, future application of personalised medicine could provide more positive outcomes to patients in dermatology. 